\title{
The Development and Validation of a Hazard Perception Test for Thai Drivers
}

2

8 Author Note

9 Correspondence should be addressed to Harry Manley, Faculty of Psychology, Chulalongkorn

10 University, Bangkok, Thailand. Email: harry.m@chula.ac.th; harrisonmanley@gmail.com

\section{Funding.}

13 This research was funded by a Mitsui Sumitomo Insurance Welfare Foundation Research Grant.

14

15 Cite As. Manley, H., Paisarnsrisomsuk, N., Hill, A. \& Horswill, M. S., (in press). The

16 Development and Validation of a Hazard Perception Test for Thai Drivers. Transportation

17 Research Part F: Psychology and Behaviour. doi:10.1016/j.trf.2020.04.011 
10 differences in computer mouse skill, mirroring crash risk differences between these groups. The

11 final 30-item version of the test, which comprised the best items from the initial test, yielded

12 novice/experienced driver differences with or without adjusting for computer mouse skill. These

13 results offer preliminary support for the validity of the latency-based test as a measure of hazard 14 perception ability in Thai drivers.

15

Keywords: Driving, Road Safety, Anticipatory Skill, Learner Driver, Experienced

\section{Drivers}




\section{Introduction}

\section{$2 \quad 1.1$ Background and Motivation}

Road traffic collisions are responsible for up to 1.35 million deaths annually and the vast

4 majority of these deaths occur in low to middle income countries (World Health Organization,

5 2018). Consequently, there is a growing recognition of the need to understand the various

6 environmental and psychological factors responsible for high fatality rates in developing

7 countries. One crash risk factor that has been studied extensively in developed countries is

8 drivers' hazard perception ability (Horswill, 2016a).

\section{$9 \quad 1.2$ Hazard Perception}

Hazard perception ability refers to a driver's skill in anticipating and detecting potential

11 road hazards (Horswill, 2016a). Drivers with good hazard perception skill are thought to build

12 effective mental models of the road environment and evaluate predictive cues to help facilitate

13 the early detection of hazards. For example, if a driver with good hazard perception is following

14 a taxi and, in scanning the road ahead, observes a pedestrian beside the road with an outstretched

15 hand, they are more likely to anticipate that the taxi may slow to pull over. As a result, they

16 might be able to respond quicker if the taxi does slow suddenly, reducing the chances of a rear-

17 end collision (for a discussion of hazard perception tests, see: Horswill, 2016b). In a typical

18 hazard perception test, drivers are presented with video clips of road scenes filmed from the

19 driver's perspective and are required to respond as quickly as possible when they detect the

20 appearance of a hazard. Hazard perception test scores have been found to correlate with both on-

21 road driving performance (Hill, Horswill, Whiting, \& Watson, 2019; Wood, Horswill, Lacherez,

22 \& Anstey, 2013) and crash involvement (Boufous, Ivers, Senserrick, \& Stevenson, 2011; Cheng,

23 Ng, \& Lee, 2011; Darby, Murray, \& Raeside, 2009; Horswill, Anstey, Hatherly, \& Wood, 2010; 
1 Horswill, Hill, \& Wetton, 2015; McKenna \& Horswill, 1999; Pelz \& Krupat, 1974; Quimby,

2 Maycock, Carter, Dixon, \& Wall, 1986; Rosenbloom, Perlman, \& Pereg, 2011). As a result,

3 hazard perception tests have been incorporated into driver licensing assessments in a growing

4 number of developed countries such as the UK, Netherlands, and Australia (Castro et al., 2014;

5 Horswill, 2016b; Wetton, Hill, \& Horswill, 2011). Notably, their implementation has had

6 discernible impact in reducing the number of road traffic accidents in some countries (Wells,

7 Tong, Sexton, Grayson, \& Jones, 2008).

8 Despite these efforts in developed countries, little is known regarding the hazard

9 perception abilities of drivers in developing countries. This lack of focus on developing countries

10 is problematic since this is where most crash fatalities occur. The research that does exist has

11 focussed on the cross-cultural transfer of latency-based hazard perception tests for developing

12 countries (Lim, Sheppard, \& Crundall, 2013; Ventsislavova et al., 2019). However, there are

13 issues regarding the validity of the tests used in this work. One strategy for evaluating hazard

14 perception test validity is to determine whether response times to hazards are slower in high risk

15 driver groups, such as novices, compared with lower risk driver groups, such as more

16 experienced drivers (Wetton et al., 2011, 2010). Lim et al., (2013) and Ventsislavova et al.

17 (2019) developed latency-based hazard perception tests using footage from both developing and

18 developed countries, but neither found significant novice/experienced driver differences in

19 hazard perception latency, regardless of test-takers' country of origin. However, Lim et al.

20 (2013) did find that drivers from a developing country (Malaysia) appeared to require a higher

21 objective level of hazardousness before defining the event as a hazard. They speculated that the

22 higher frequency of traffic conflicts in developing countries may cause experienced drivers to

23 become desensitized to hazardous situations and take longer to acknowledge them as hazards, 
1 without necessarily being worse at hazard perception. Similarly, Ventsislavova et al. (2019)

2 found that Chinese drivers responded to fewer hazards than UK drivers, as well as responding to

3 them more slowly, which the authors suggested might reflect a criterion bias (their Chinese

4 sample were less sensitive to, or accepting of, hazards overall). This might imply that even a

5 latency-based hazard perception test that was valid for UK drivers, nonetheless might not be

6 appropriate for drivers from developing countries due to cultural differences in how dangerous

7 an event has to be before drivers consider classifying it as a hazard.

8 Considering these difficulties in validating hazard perception tests across cultures,

9 Ventsislavova and colleagues suggested that future efforts should focus on alternative tests

10 without a latency-based response mode (Ventsislavova et al., 2019). However, we propose that

11 it is premature to abandon attempts to develop a latency-based hazard perception tests for

12 developing countries, because there are as-yet untried strategies that may address the validity

13 problems reported to date.

14 First, Wetton et al. (2011) proposed a solution to the criterion bias issue involving

15 changes in terminology in the test instructions. Instead of asking participants to respond to

16 "hazards", they instead asked participants to respond when they anticipated "traffic conflicts"

17 and the term "hazard" was not mentioned in the test instructions. Traffic conflicts were defined

18 as any event in which the car with the camera has to brake or change course to avoid a collision

19 with another road user. Test-takers were asked to use a computer mouse to click on any road user

20 that was likely to become involved in a traffic conflict with the camera car. While test-takers can

21 argue that they did not respond to an event because they did not believe it of sufficient risk to be

22 classified as a hazard (i.e. they can claim that the event "wasn't a hazard" to them), it is harder

23 for them to argue that they did not respond to a traffic conflict because it "wasn't a traffic 
1 conflict" to them. This is because, compared with the definition of a hazard, the definition of a

2 traffic conflict is less subjective (i.e. the camera car either has to take evasive action to avoid a

3 collision or it does not). Tests employing the "traffic conflict" instruction strategy have been

4 found to yield both novice/experience differences (Hill et al., 2019; Wetton et al., 2011) and

5 relationships with crash involvement (Horswill et al., 2015).

6 Second, the absence of novice/experienced differences reported by Lim et al. (2013) may

7 have been due to the relatively low post-license experience of the drivers that were classified as

8 experienced. The experienced groups (both Malaysian and UK samples) were under 22 years of

9 age and had, on average, fewer than 50 months of driving experience. Although Ventsislavova et

10 al., (2019) examined a larger sample of drivers with a more pronounced distinction between

11 novice and experienced drivers, they used a button press response mode in which participants did

12 not indicate the on-screen location of the hazard. Other latency-based tests (Wetton et al., 2011,

13 2010) have instead used a computer mouse response mode in which participants have to click on

14 the correct on-screen location of the hazard. This has been argued to potentially reduce

15 measurement error (Wetton et al., 2011) by excluding situations when individuals respond

16 quickly but to the wrong event.

17 We propose the development of a latency-based hazard perception test for a developing

18 country that (1) uses "traffic conflict" instructions that do not include the term "hazard", (2) uses

19 a response mode requiring the on-screen location of the source of the traffic conflict to be

20 specified, and (3) is evaluated using novice/experienced groups that are more differentiated in

21 average experience.

\section{$22 \quad 1.3$ Present Study}


The central focus of the present research is to develop and validate a latency-based measure to assess the hazard perception abilities of drivers in Thailand. Thailand is a developing

3 country with a high rate of road fatalities per capita (32.7 per 100,000 drivers; World Health

4 Organization, 2018) and with $77.5 \%$ of crashes attributed to "human error" (World Health

5 Organization, 2015). Established hazard perception tests that show road environments from

6 developed countries may be problematic for use with Thai drivers because of the substantially

7 different driving environment in Thailand (e.g., relative risk, road layout, and hazard types). For

8 example, in Thailand, there are more interactions with two-wheeled vehicles, the predictive

9 signals from other road users are less consistent (e.g., road users are less likely to indicate before

10 turning), and right of way is infrequently observed at pedestrian crossings. Consequently, the

11 current paper sets out to develop a Thailand-specific latency-based hazard perception test

12 designed to examine Thai drivers' ability to anticipate hazards.

13 As previously discussed, we used a version of the instructions developed by Wetton et al.

14 (2011), in which participants are asked to respond to "traffic conflicts", and the term "hazard" is

15 not mentioned. We also used a computer mouse response mode in which test-takers had to

16 identify the correct location of relevant road users on the screen.

17 We examined the validity of the hazard perception test by determining whether it could

18 discriminate between high- and lower-risk driver groups. Consistent with developed countries,

19 the relative crash risk of novice drivers is considerably higher than experienced drivers in

20 Thailand (Tanaboriboon \& Satiennam, 2005) and thus, in the present research, we compared the

21 hazard perception test performance of novice and experienced drivers. If experienced drivers

22 outperform novices, then this evidence can be considered as supporting the case for test validity.

\section{Methods}


All data, analysis scripts, and video illustration of the experimental setup are available on the Open Science Framework (https://osf.io/cp9bg).

\subsection{Participants} minutes and participants were compensated with 400 baht ( \$13 USD) or course credit. A

6 recruitment poster advertising the study was distributed online, via email, and on social media.

7 All participants provided written informed consent. Four participants were excluded from the

8 final analysis either because they (1) did not understand the instructions $(n=2)$, (2) reported

9 difficulty viewing the stimuli $(n=1)$, or $(3)$ responded on fewer than $50 \%$ of trials $(n=1)$.

10 Consequently, our final sample included 48 novice (33 females, 15 males) and 87 experienced

11 (46 females, 41 males) drivers (see Table 1 for demographic statistics for each group). Consistent

12 with previous research (Crundall, 2016; Kinnear, Kelly, Stradling, \& Thomson, 2013), we

13 defined experienced drivers as those holding a full driving license for a minimum of three or

14 more years and novice drivers as those who held a license for fewer than three years.

\section{$15 \quad 2.2$ Measures 1}

\subsubsection{Hazard perception test: development of the initial item-pool. Raw footage of}

17 driving scenes was gathered (primarily) from dashboard cameras mounted inside two cars driven

18 by members of the research team around rural and urban (Bangkok) areas of Thailand. For all

19 footage, we ensured that the image captured approximately the driver's point of view. In addition

20 to the footage recorded using our own cameras, we also invited people via social media (e.g.,

21 members of a car camera club in Thailand) to submit video clips of potential traffic conflicts they

\footnotetext{
1 After completing the hazard perception test, participants also completed a nine-clip hazard prediction test whereby they were asked to predict what would happen next in a complex visual scene after the image is occluded. We added this test as an exploratory measure and do not report further details here because the occlusion time was potentially problematic for some of the clips.
} 
1 had recorded. Contributors provided consent for their footage to be used in the research. After

2 viewing all footage, we identified 243 potential traffic conflicts.

2.2.2 Hazard perception test: evaluation of hazard scenes. In line with best practice guidelines for the development of hazard perception footage (Wetton et al., 2011), two trained researchers then rated each of the 243 hazard clips featuring potential traffic conflicts according to five criteria: (1) Image quality did not appear pixelated; (2) the road ahead had a clear view without reflections, glares, bends, water drops, or vibrations that would disturb the test takers' perceptions of objects on the road; (3) the video clips contained subtle anticipatory predictive cues of each impending traffic conflict; (4) each traffic conflict was distinct and had at least a three-to-four second gap from any other traffic conflict; and (5) the traffic conflicts required drivers to respond by stopping, slowing down, or changing direction. The 113 traffic conflicts that were judged to meet these criteria sufficiently were then reviewed by a third rater (first author) and any that were considered ambiguous or inappropriate were removed, leaving a final sample of 77 traffic conflicts in the item pool.

\subsubsection{Hazard perception test: implementation. Each video clip was edited to be} between $13 \mathrm{~s}$ and $30 \mathrm{~s}$ in length $(M=23.74 \mathrm{~s})$ and there was a total of 55 unique clips, each featuring one or more traffic conflicts. Thirty-seven clips contained a single unique traffic conflict, 14 clips contained two traffic conflicts, three clips contained three traffic conflicts, and one clip contained four unique traffic conflicts. Consequently, there was a total of 77 separate identifiable traffic conflicts in the initial test. To identify accurate hits, we used custom software to manually define a target region containing the road user that was source of the traffic conflict (taking into account any minor inaccuracies in mouse click location). This region was updated on each frame to track the target as needed. These target regions were not visible to participants. To 
1 check that the target regions were appropriate, we reviewed all missed targets for the first 40

2 participants, correcting the accepted target regions as needed. Reaction times to each traffic

3 conflict were calculated from the first point when the traffic conflict could potentially be

4 responded to (i.e. from the onset of the anticipatory cues).

2.2.4 Mouse Skills Test. Participants completed a mouse skills test to assess their

6 proficiency controlling the on-screen pointer. The test required participants to use the mouse to

7 click on a series of black rectangles (approximately the same size as the targets in the main test),

8 which appeared, one at a time, at pseudorandom locations on the screen, with a delay of between

92 to 5 seconds between each rectangle. Participants were instructed to click on the rectangle as

10 fast as possible when it appeared, then to move their mouse pointer back to the bottom of the

11 screen. The participant's mouse skills test score was their mean response time to click on the

12 rectangles.

\section{$13 \quad 2.3$ Procedure}

All tests were displayed on a 17" LCD screen using custom software designed for hazard

15 perception tests. At the start of the experiment, participants provided information about their

16 demographic details and driving history. Participants then completed the mouse skills test and

17 watched a video explaining the hazard perception test. The instruction video presented

18 instructions and audio in Thai and featured example road hazards displayed from a driver's

19 perspective and provided the following definition of a traffic conflict: "A traffic conflict is a

20 situation where your vehicle is on course to hit another road user. If your vehicle needs to slow

21 down or change course to prevent a crash, then there is a traffic conflict." We instructed

22 participants to anticipate and respond to each of the traffic conflicts as quickly as possible. At the

23 end of the instruction video, we asked participants to confirm that they understood the 
1 instructions before continuing to the main hazard perception test 2 . A video of all test procedures

2 is available on the Open Science Framework (OSF; https://osf.io/8xcqg). Participants then

3 completed the main hazard perception test. To prevent order effects, the clips were presented in a

4 different computer-generated random sequence for each participant. To minimize fatigue,

5 participants were given the opportunity to take a short, self-paced break after every 18 trials.

\section{$6 \quad 2.4$ Analysis}

7 In line with previous approaches (Wetton et al., 2010), missing values for the hazard

8 perception test were replaced with the maximum response time for the conflict as a penalty for

9 not responding. Reaction time scores for the hazard perception test were calculated by averaging

10 the standardized response times across all hazards (raw scores were converted into z-scores using

11 the overall sample mean and $S D$ for each hazard). This process was used because the hazards

12 varied in duration and, without standardization, some hazards might exert a greater influence on

13 the final mean score than others. The overall standardized score was then converted back to an

14 overall response time (measured in seconds) using the mean and $S D$ across all hazards and all

15 participants. In our main analysis, we examined differences in experienced and novice drivers'

16 hazard perception time, controlling for participants' mouse skills. We removed the effect of

17 individual differences in mouse skills by subtracting each individual's mouse skills response

18 time from their hazard perception test responses to yield a hazard perception time3. We then

19 compared novice/experienced differences use a Welch's independent samples t-test. Cohen's $d$ is

20 reported as a measure of effect size with $0.2,0.5$ and 0.8 reflecting small, medium, and large

2 The following text was displayed: "Did you understand the instructions?". To respond, participants had two choices: (1) click on the "NO" button to play the instruction video again; or (2) click on the "YES" button to begin the test.

3 An alternative analysis is to conduct an ANCOVA, controlling for mouse skills as a covariate. However, we were unable to perform this test due to violations of assumptions. 
1 effect sizes, respectively. Processing of data, visualisation, and all statistical tests were

2 performed using Jamovi and $R$; analysis scripts, data and codebook are available on the OSF

3 (https://osf.io/cp9bg).

5 Compared to the novice drivers, the experienced group were older, more experienced,

6 drove further and for longer each week (see Table 1), and had a similar educational background

7 (see Table 2).

8 Table 1

9 Descriptive statistics for study sample.

\begin{tabular}{|c|c|c|c|c|c|}
\hline & $\begin{array}{r}\text { Age } \\
\text { (years) }\end{array}$ & $\begin{array}{r}\text { Driving } \\
\text { experience } \\
\text { (years) }\end{array}$ & $\begin{array}{l}\text { Driving distance } \\
\text { (KM per week) }\end{array}$ & $\begin{array}{l}\text { Driving time } \\
\text { (Hrs per week) }\end{array}$ & $\begin{array}{r}\text { Mouse skills } \\
\text { (s) }\end{array}$ \\
\hline $\begin{array}{l}\text { Novice } \\
(N=48)\end{array}$ & $\begin{array}{l}21.12 \\
(3.80)\end{array}$ & $\begin{array}{r}1.25 \\
(0.58)\end{array}$ & $\begin{array}{r}100.79 \\
(172.36)\end{array}$ & $\begin{array}{r}7.26 \\
(11.81)\end{array}$ & $\begin{array}{r}0.90 \\
(0.14)\end{array}$ \\
\hline $\begin{array}{l}\text { Experienced } \\
(N=87)\end{array}$ & $\begin{array}{r}36.08 \\
(12.82)\end{array}$ & $\begin{array}{r}14.49 \\
(11.91)\end{array}$ & $\begin{array}{r}224.87 \\
(241.50)\end{array}$ & $\begin{array}{r}12.59 \\
(10.95)\end{array}$ & $\begin{array}{r}1.04 \\
(0.22)\end{array}$ \\
\hline $\begin{array}{l}\text { Test of mean } \\
\text { difference } \\
p \text {-value } \\
\text { Cohen's } d\end{array}$ & $\begin{array}{r}<.001 \\
1.417\end{array}$ & $\begin{array}{r}<.001 \\
1.382\end{array}$ & $\begin{array}{r}<.001 \\
0.565\end{array}$ & $\begin{array}{r}.014 \\
0.474\end{array}$ & $\begin{array}{r}<.001 \\
0.771\end{array}$ \\
\hline
\end{tabular}


$1 \quad$ Table 2

2 Frequency of study sample by highest education level obtained.

\begin{tabular}{lcccccc}
\hline & Elementary & $\begin{array}{c}\text { High } \\
\text { School }\end{array}$ & $\begin{array}{c}\text { Vocational } \\
\text { College }\end{array}$ & Diploma & Bachelor & Masters \\
\hline Novice & 0 & 1 & 0 & 0 & 45 & 2 \\
Experienced & 2 & 3 & 5 & 0 & 51 & 26 \\
\hline
\end{tabular}

3

$4 \quad 3.1$ Hazard Perception Test: Initial 77-item test

$5 \quad$ We conducted a Welch's independent samples t-test to examine whether there were any

6 differences in hazard perception times as a function of driver group. This indicated there was a

7 significant effect of driver group $(t(115)=3.14, p=.002, d=.531)$ on hazard perception

8 response times (see Figure 1). Experienced drivers $(M=2.84, S D=.60)$ were, on average, 296

9 ms faster to detect traffic conflicts than novice drivers, $(M=3.13, S D=.48)$. The initial hazard

10 perception test including all 77 traffic conflicts had good internal consistency, Cronbach's alpha

$11=.93$; item-rest correlation $M=.42, S D=.13$, minimum $=0.07$, maximum $=0.64$. Bivariate

12 correlations between hazard perception test scores and other key variables are presented in Table 133. 


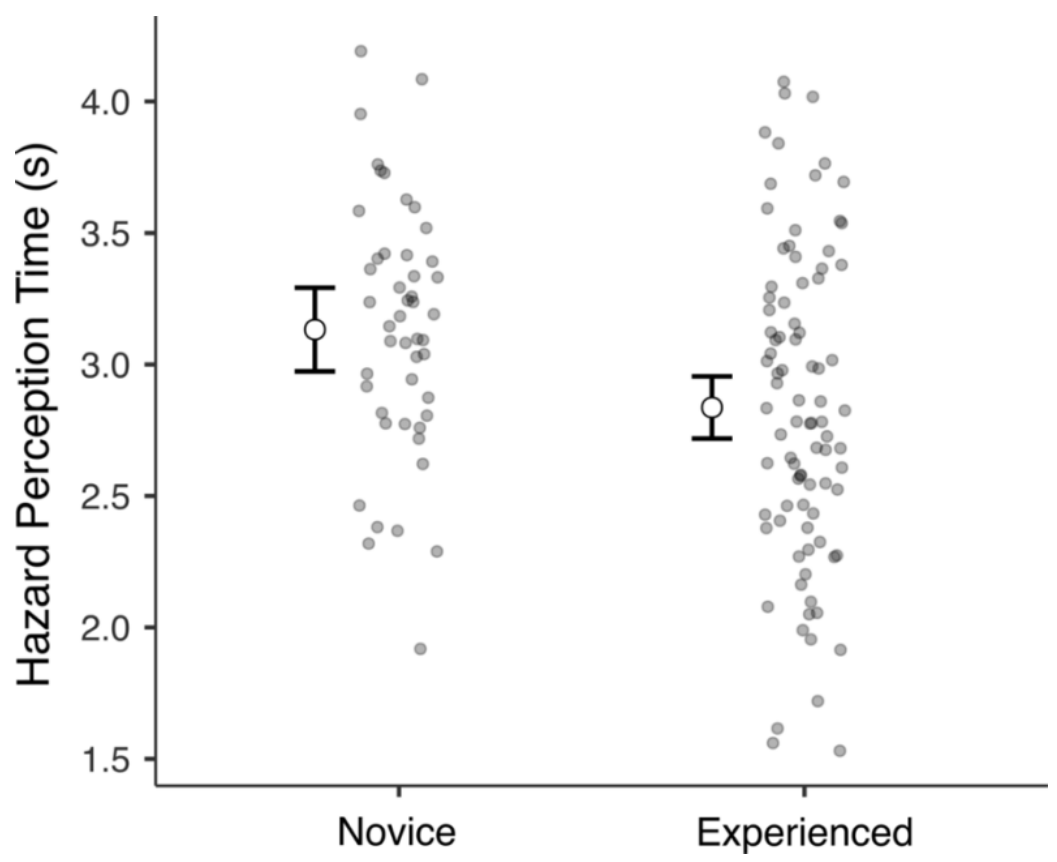

2 Figure 1. Hazard perception times (estimated marginal means) as a function of driver group for

3 the initial 77-item test. Error bars indicate 95\% confidence interval of the mean; individual data

4 points are plotted in grey.

\section{$5 \quad$ 3.2 Hazard Perception Test: Final 30-item test}

Having first shown that the entire 77-item pool of clips could successfully distinguish

7 between novice and experienced drivers, we next sought to identify an appropriate subset of test

8 items that could be used in future hazard perception research in Thailand. Group differences for

9 each traffic conflict were examined using a series of Welch's $t$-tests. Overall, experienced drivers

10 had faster hazard perception times than novice drivers for 51 of the 77 traffic conflicts. After

11 excluding all trials with low hit rates (i.e., where fewer than $70 \%$ of participants responded to the

12 target) traffic conflicts were rank-ordered according to their effect sizes to create a final 30-item

13 test that could be used in future research (this number of items was chosen in order to yield a test

14 of practical duration for research purposes). 
The novice/experienced difference in response time for the final 30-item test was

2 statistically significant $(t(107)=4.34, p<.001, d=.780)$; experienced $(M=3.34, S D=.81)$

3 drivers were, on average, $604 \mathrm{~ms}$ faster to detect traffic conflicts on the 30-clip pool of traffic

4 conflicts than novice $(M=3.94, S D=.71)$ drivers, (see Figure 2). The final test also had good

5 internal consistency, Cronbach's alpha $=.87$; item-rest correlation $M=.45, S D=.11$, minimum

$6=0.25$, maximum $=0.57$.

7 Notably, the novice/experienced group difference was also present when entering the

8 response times for the hazard perception test without controlling for mouse skill ability,

9 suggesting this test is somewhat robust to differences in mouse proficiency when the data are

10 interpreted at the group level. A Welch's t-test $(t(114)=3.25, p=.002)$ confirmed this

11 difference, with experienced drivers responding an estimated $456 \mathrm{~ms}$ faster than novice drivers

12 (Cohen's $d=0.553)$.

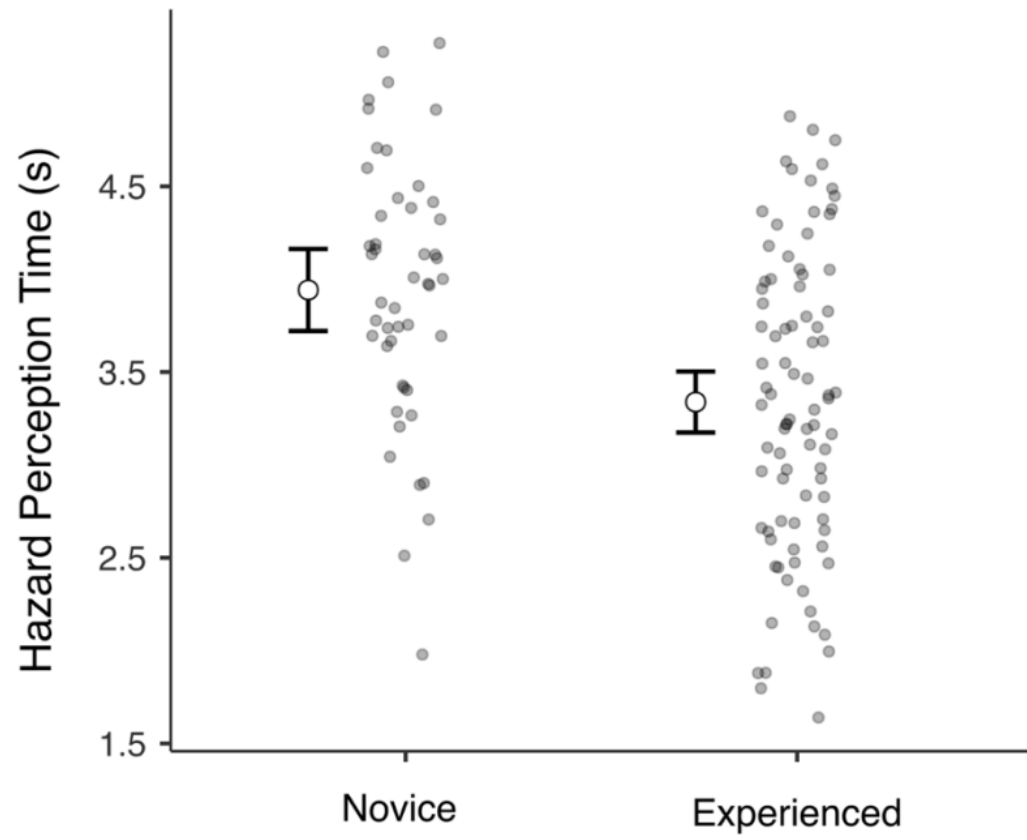

14 Figure 2. Hazard perception times as a function of driver group for the 30-clip test. Error bars

15 indicate $95 \%$ confidence interval of the mean; individual data points are plotted in grey 
$1 \quad$ Table 3

2 Zero-order correlations for key variables.

\begin{tabular}{lcccrr}
\hline & 1 & 2 & 3 & 4 & 5 \\
\hline 1. Hazard Perception Time -77 items & - & & & & \\
2. Hazard Perception Time -30 items &. $\mathbf{9 3}$ & - & & & \\
3. License Length (years)* & $\mathbf{- 1 7}$ & $\mathbf{- . 3 2}$ & - & & - \\
4. Mouse Skills & .03 & .01 & $\mathbf{. 4 5}$ & & \\
5. Age * & -.02 & -.20 & $\mathbf{9 0}$ & $\mathbf{. 5 0}$ & - \\
Mean & 2.94 & 3.55 & 9.79 & 0.99 & 30.80 \\
SD & 0.57 & 0.82 & 11.50 & 0.20 & 12.70 \\
Skewness & -0.16 & -.20 & 1.41 & 0.91 & 1.05 \\
Kurtosis & -0.34 & -.62 & 0.89 & 0.68 & -0.08 \\
\hline
\end{tabular}

$3 \quad$ Note. Significant correlations $(p<.05)$ are indicated in bold. *Spearman's rho reported for

4 correlations with license length and age.

\section{Discussion}

Previous efforts to construct latency-based hazard perception tests in developing

7 countries have met with little success, with test performance failing to discriminate between

8 experienced and novice drivers (Lim et al., 2013; Ventsislavova et al., 2019). In the present study

9 we show that a variant of the latency-based test that requires responses to the correct target

10 location rather than simple button-press response times, is a feasible approach. As evidence for

11 the test's validity, test scores could discriminate between the performance of samples of novice

12 and experienced Thai drivers. We found that experienced drivers detected traffic conflicts an

13 estimated $604 \mathrm{~ms}$ faster than novice drivers on the 30-clip version of the test, consistent with the

14 proposal that the test is a valid measure of hazard perception ability. For a car travelling at only 
$140 \mathrm{mph}$, a $604 \mathrm{~ms}$ delay in reaction time to detect a hazard translates to approximately $11 \mathrm{~m}$ in

2 road distance travelled; a distance that would likely be a contributing factor in determining

3 whether a driver would successfully avoid or collide with another road user involved in a

4 developing traffic conflict.

5 In our variant of the hazard perception test, participants are required to control the cursor

6 and click on a target region (i.e., another road user involved in a likely traffic conflict). The

7 benefit of this approach is that it ensures all reaction times reflect target hits. However, a

8 drawback is that reaction times are affected by individual differences in computer mouse skills,

9 which introduces variation unlikely to map onto differences in driving ability. In the current

10 study, we removed this noise by subtracting reaction times on the mouse skills test. We observed

11 significant differences between novice and experienced drivers on this measure (presumably as a

12 result of age-related cohort differences, see Table 3). Notably, the mean group difference in

13 mouse ability was larger for the current sample $(M=148 \mathrm{~ms})$ compared to an Australian sample

$14 \quad(M=112 \mathrm{~ms}$; Wetton et al., 2012), suggesting that individual differences in mouse skill may exert

15 a greater influence on hazard perception response times on the Thai test compared to the effect

16 they have in Western samples. This might be a result of greater individual difference variation in

17 computer mouse skills in Thai participants and emphasises the potential importance of

18 accounting for these factors when studying hazard perception abilities of individuals in

19 developing countries. Note however that the final 30-item test did not require any adjustment for

20 mouse skill to reveal group-level novice/experienced differences in hazard perception. One

21 possible explanation for this is that the traffic conflicts included in the 30 -item test may have

22 been more amenable to being anticipated by more skilled drivers (i.e. they were better measures 
1 of hazard perception skill) and hence the noise from individual differences in mouse skills had

2 less influence.

\section{$3 \quad 4.1$ Implications and Future Directions}

$4 \quad$ One issue we encountered when selecting clips was that many of the naturally occurring

5 hazards appeared both abruptly and unexpectedly, where there would be limited scope for drivers

6 with superior hazard perception ability to be able to use their skills to detect and respond earlier

7 (Ventsislavova et al., 2019). We excluded hazards that could not reasonably be predicted because

8 we were interested in developing a test that assessed drivers' ability to anticipate hazards.

9 However, one potential direction for future research would be to understand how driving

10 experience influences broader preventative driving behaviours in order to minimise exposure to

11 abrupt and unexpected events. For example, it is unclear whether repeated past experiences of

12 near misses and accidents would desensitise Thai drivers and increase their tolerance to risk or

13 whether they adapt the way they drive (i.e., by learning to avoid placing their vehicle in high-risk 14 situations).

15 Despite the significant differences in the mean reaction times between novice and

16 experienced drivers, we suggest that further work is warranted before such a test can be used as

17 part of a formal driver licensing test in Thailand. For instance, we would recommend a larger-

18 scale study to determine if the test scores can predict crash involvement directly (as per Horswill

19 et al., 2015). If such a relationship was found, then this would create a strong case for the road

20 safety benefits of introducing a licensure test. If Thai drivers had to pass a hazard perception test

21 to obtain their licence, hence mandating a minimum standard of hazard perception skill, then this

22 would be expected to (1) provide an incentive for new drivers to improve their hazard perception

23 ability through additional training and (2) screen out drivers who do not meet the standard. This 
1 may result in reduced crash rates, as has been documented in other countries (Wells et al., 2008).

2 Nevertheless, the present test was developed as research tool, and it is therefore sufficient that it

3 has been demonstrated to provide meaningful data at the group level, rather than at the level of

4 the individual test-taker. However, the fact that this has been achieved with Thai-specific traffic

5 conflict clips suggests that the larger-scale investment necessary to produce a hazard perception

6 test suitable for licensure purposes in Thailand would be justified.

7 Licensing policy implications aside, there are many potential applications of the test as a

8 research tool. For example, it could be used to understand how specific factors (in addition to

9 driving experience) affect hazard perception ability in Thai drivers. For instance, there is

10 considerable heterogeneity in the degree of formal driver training that is undertaken by Thai

11 drivers and the impact of this on hazard perception is unknown. Understanding which factors are

12 important may be useful to help target broader policy interventions.

13 It is also important that future research directs greater attention towards hazard perception

14 ability as it relates to vulnerable road users. This is necessary because only $12 \%$ of fatalities in

15 Thailand involve occupants of 4-wheeled vehicles, as opposed to $48 \%$ in Europe, and hence a

16 much greater proportion of victims are vulnerable road users such as pedestrians and users of 2-

17 or 3-wheeled vehicles (World Health Organization, 2018). Thus, in countries like Thailand, it is

18 particularly critical to understand how these vulnerable road users are perceived by car drivers

19 and how hazard perception skill might differ as a function of the type of vehicle driven.

20 Further, given the paucity of research on the cognitive-affective-motivational processes

21 of Thai drivers, there is a pressing need for efforts to examine how psychological factors are

22 related to crash risk. Specifically, understanding how broader aspects of situation awareness (i.e.,

23 perception, comprehension, and prediction of hazards) and decision-making processes interact 
1 with sociocultural factors will be important to consider. While hazard perception ability is only

2 one contributor to road safety, it is likely to interact with other factors, such as the decision to

3 drive safely or dangerously (e.g., speeding) and the constraints (or lack of) to behaviour via

4 traffic laws and enforcement.

\section{$5 \quad 4.2$ Conclusion}

6 The high road traffic fatality rate in Thailand is a source of considerable social (as well as

7 financial) cost, yet relatively little is understood regarding the psychological processes of Thai

8 drivers. In a step to address this, we successfully developed a Thai version of a latency-based

9 hazard perception test. The upcoming challenge is to apply this test to further our understanding

10 of drivers who are at the greatest crash risk and use this knowledge to develop interventions to

11 improve road safety for Thai drivers. 


\section{Acknowledgements}

2 We thank the following individuals for their contributions: Somboon Jarukasemthawee (general

3 assistance, video footage), Kullaya Pisitsungkagarn (general assistance), Wachrapong

4 Foophrateepsiri (compiling footage, data collection), and Trin Savejnarong, Pattramon

5 Pornsukjantra, and Ponpicha Lerthirunvibul (data collection). 


\section{References}

Boufous, S., Ivers, R., Senserrick, T., \& Stevenson, M. (2011). Attempts at the Practical OnRoad Driving Test and the Hazard Perception Test and the Risk of Traffic Crashes in Young Drivers. Traffic Injury Prevention, 12(5), 475-482. https://doi.org/10.1080/15389588.2011.591856

Castro, C., Padilla, J. L., Roca, J., Benítez, I., García-Fernández, P., Estévez, B., ... Crundall, D. (2014). Development and Validation of the Spanish Hazard Perception Test. Traffic Injury Prevention, 15(8), 817-826. https://doi.org/10.1080/15389588.2013.879125

Cheng, A. S. K., Ng, T. C. K., \& Lee, H. C. (2011). A comparison of the hazard perception ability of accident-involved and accident-free motorcycle riders. Accident Analysis \& Prevention, 43(4), 1464-1471. https://doi.org/10.1016/j.aap.2011.02.024

Crundall, D. (2016). Hazard prediction discriminates between novice and experienced drivers. Accident Analysis and Prevention, 86, 47-58. https://doi.org/10.1016/j.aap.2015.10.006

Darby, P., Murray, W., \& Raeside, R. (2009). Applying online fleet driver assessment to help identify, target and reduce occupational road safety risks. Safety Science, 47(3), 436-442. https://doi.org/10.1016/j.ssci.2008.05.004

Hill, A., Horswill, M. S., Whiting, J., \& Watson, M. O. (2019). Computer-based hazard perception test scores are associated with the frequency of heavy braking in everyday driving. Accident Analysis \& Prevention, 122, 207-214. https://doi.org/10.1016/j.aap.2018.08.030

Horswill, M. S. (2016a). Hazard Perception in Driving. Current Directions in Psychological Science, 25(6), 425-430. https://doi.org/10.1177/0963721416663186 
1 Horswill, M. S. (2016b). Hazard perception tests. In D. L. Fisher, J. K. Caird, W. Horrey, \& L. Trick, Eds.). The handbook of teen and novice drivers: Research, practice, policy, and directions (pp 439-450) Boca Raton, FL: CRC Press.

Horswill, M. S., Anstey, K. J., Hatherly, C. G., \& Wood, J. M. (2010). The crash involvement of older drivers is associated with their hazard perception latencies. Journal of the International Neuropsychological Society, 16(5), 939-944. https://doi.org/10.1017/S135561771000055X

Horswill, M. S., Hill, A., \& Wetton, M. A. (2015). Can a video-based hazard perception test used for driver licensing predict crash involvement? Accident Analysis \& Prevention, 82, 213219. https://doi.org/10.1016/j.aap.2015.05.019

Kinnear, N., Kelly, S. W., Stradling, S., \& Thomson, J. (2013). Understanding how drivers learn to anticipate risk on the road: A laboratory experiment of affective anticipation of road hazards. Accident Analysis and Prevention, (50), 1025-1033. https://doi.org/10.1016/j.aap.2012.08.008

Lim, P. C., Sheppard, E., \& Crundall, D. (2013). Cross-cultural effects on drivers' hazard perception. Transportation Research Part F: Traffic Psychology and Behaviour, 21, 194206. https://doi.org/10.1016/j.trf.2013.09.016

McKenna, F. P., \& Horswill, M. S. (1999). Hazard perception and its relevance for driver licensing. Journal of the International Association of Traffic and Safety Sciences, 23(1), $26-41$.

Pelz, D. C., \& Krupat, E. (1974). Caution profile and driving record of undergraduate males. Accident Analysis \& Prevention, 6(1), 45-58. https://doi.org/10.1016/00014575(74)90015-3 
1 Quimby, A. R., Maycock, G., Carter, I., Dixon, R., \& Wall, J. G. (1986). Perceptual abilities of accident involved drivers (Report No. RR27) (No. RR27). Retrieved from http://www.trl.co.uk/reports-publications/trl-reports/report/?reportid=5668

Rosenbloom, T., Perlman, A., \& Pereg, A. (2011). Hazard perception of motorcyclists and car drivers. Accident Analysis \& Prevention, 43(3), 601-604. https://doi.org/10.1016/j.aap.2010.08.005

Tanaboriboon, Y., \& Satiennam, T. (2005). Traffic Accidents in Thailand. IATSS Research, 29(1), 88-100. https://doi.org/10.1016/s0386-1112(14)60122-9

Ventsislavova, P., Crundall, D., Baguley, T., Castro, C., Gugliotta, A., Garcia-Fernandez, P., ... Li, Q. (2019). A comparison of hazard perception and hazard prediction tests across China, Spain and the UK. Accident Analysis and Prevention, 122, 268-286. https://doi.org/10.1016/j.aap.2018.10.010

Wells, P., Tong, S., Sexton, B., Grayson, G., \& Jones, E. (2008). Cohort II: A study of learner and new drivers: Vol. 1 (Road Safety Research Report No. 81). London: Department for Transport

Wetton, M. A., Hill, A., \& Horswill, M. S. (2011). The development and validation of a hazard perception test for use in driver licensing. Accident Analysis and Prevention, 43(5), 1759-1770. https://doi.org/10.1016/j.aap.2011.04.007

Wetton, M. A., Horswill, M. S., Hatherly, C., Wood, J. M., Pachana, N. A., \& Anstey, K. J. (2010). The development and validation of two complementary measures of drivers' hazard perception ability. Accident Analysis and Prevention, 42(4), 1232-1239. https://doi.org/10.1016/j.aap.2010.01.017 
1 Wood, J. M., Horswill, M. S., Lacherez, P. F., \& Anstey, K. J. (2013). Evaluation of screening tests for predicting older driver performance and safety assessed by an on-road test. Accident Analysis and Prevention, (50), 1161-1168. https://doi.org/10.1016/j.aap.2012.09.009

5 World Health Organization. (2015). Global status report on road safety 2015. Geneva. http://www.who.int/violence_injury_prevention/road_safety_status/2015/en/

7 World Health Organization. (2018). Global status report on road safety 2018. Geneva. http://www.who.int/violence_injury_prevention/road_safety_status/2018/en/ 